\title{
Shape Optimisation of Displaced Enhancement Devices for Heat Transfer Augmentation by Inverse Problem Approach Applied to Infrared Images
}

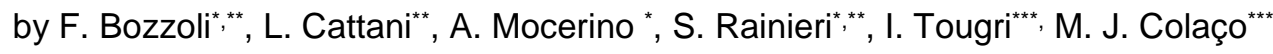 \\ * Department of Engineering and Architecture, University of Parma, Parco Area delle Scienze 181/A, 43121, \\ Parma, Italy, fabio.bozzoli@unipr.it \\ ** SITEIA.PARMA Interdepartmental Centre, University of Parma, Parco Area delle Scienze 181/A, 43121, \\ Parma, Italy \\ *** Federal University of Rio de Janeiro, Department of Mechanical Engineering, Cx. Postal 68503, Rio de Janeiro, \\ RJ, 21941-972, Brazil
}

\begin{abstract}
Displaced enhancement devices are inserts, positioned inside pipes, with the aim of increasing the heat transfer rate. The actual work presents an original application of an inverse analysis technique to experimental infrared temperature data with the aim of estimating the local convective heat flux for forced convection flow in pipe when this kind of devices is present and, thanks to these results, with the aim of optimising insert geometry. In particular, butterfly-shaped inserts are investigated in terms of local and global thermal performances.
\end{abstract}

\section{Introduction}

The heat transfer enhancement techniques represent an important research topic in the field of heat transfer. In many industrial fields, such as in the food, chemical and pharmaceutical industries, engineers have then been striving for techniques producing enhanced heat transfer coefficients, accompanied by reduced pumping power requirements.

The techniques of increase of the heat transfer can be divided into active techniques that require a mechanical aid or electrostatic fields and passive techniques that do not require an external power. Among the passive technique, there is the insertion of devices in the main flow [1]. This enhancement technique results particular attractive for the low cost, the rapid installation and the easy maintenance. Displaced enhancement devices are a particular kind of inserts that leaves the heated surface substantially unaltered and changes the fluid flow near the pipe wall increasing the transferred energy. They "displace" the fluid from the heated or cooled surface of the duct and mix it with bulk fluid from the core flow [2]. Many studies were carried out on different types of displaced enhancement devices (e.g., metal grids, disks, cones, static mixers, crowns and balls). [2-4] are the most remarkable.

Displaced enhancement devices have been mainly discussed in the available scientific literature by considering only the heat transfer performance averaged over the tube heat transfer surface area. This approximated approach, which is acceptable for many applicative cases, comes from the practical difficulty of local measuring heat flux on the internal wall surface of a pipe. However, these inserts usually induce significant spatial variation of local thermal performances and the quantitative knowledge of this aspect, in some industrial applications, is of primary importance.

The present work aims to start to fill this gap by adopting an original experimental procedure to estimate local convective heat flux in pipes with this type of inserts. The estimation procedure hereby presented is based on the solution of the Inverse Heat Conduction Problem (IHCP) within the wall domain by following a formulation that adopts, as input data, the infrared temperature maps of the external pipe wall [5]. This approach overcomes the experimental problem of monitoring heat transfer on the internal wall of the tube. However, it presents some complications because IHCPs are illposed and, consequently, they are very sensitive to small perturbations in the input data. In order to bypass the illposedness of inverse problems, many techniques based on the processing of the experimental data have been suggested and validated in literature (e.g., regularization techniques, methods based on filtering proprieties, the function specification methods, probabilistic methods and iterative methods). In the present work in order to estimate the local convective heat flux at the fluid-internal wall interface it was adopted the Tikhonov regularisation method [6] that is one of the most commonly employed regularization techniques.

In particular, a butterfly-shaped device was tested at different fluid flow velocities in terms of global and local thermal performances in order to find, given a specific application, the optimal geometry.

\section{Experimental setup}

A picture of the considered butterfly-shaped device is reported in Fig. 1: the device is characterised by two "wings" and two "indentions"; it presents a maximum diameter of $13 \mathrm{~mm}$. The inserts were positioned inside a stainless steel tube with an internal diameter of $13 \mathrm{~mm}$, and a wall thickness of $1.0 \mathrm{~mm}$. The pipe was three meters long and the butterflyshaped devices were positioned at a distance of $300 \mathrm{~mm}$ one from the other in other to not override the effects of two 
consequential inserts: they were maintained in a prescribed position thanks to a central stick, as it is possible to see from Fig. 1.


Fig. 1: Picture of the inserts, sketch of the insert and sketch of the pipe with the insert (from left to right);

The working fluid, water, entered the test section equipped with stainless-steel fin electrodes, which were connected to a power supply. This setup allowed investigating the heat transfer performance of the tube under the prescribed condition of uniform heat flux generated by the Joule effect in the wall. The whole length of the heat transfer section was thermally insulated to minimize the heat transfer to the environment. The inlet temperature was measured by a thermocouple probe placed on the tube's wall upstream the starting heating section.

A small portion of the external tube wall, around the position of the insert, was made accessible to a thermal imaging camera by removing the thermally insulating layer, and it was coated with a thin film of high emissivity paint. This thin paint layer changes the surface emissivity without affecting the heat conduction problem in the tube wall. The surface temperature distributions were acquired by means of a FLIR SC7000 unit, with a $640 \times 512$ pixel detector array. Its thermal sensitivity, as reported by the instrument manufacturer, is $20 \mathrm{mK}$ at $303 \mathrm{~K}$, while its accuracy is $\pm 1 \mathrm{~K}$. A schematic view of the infrared thermographic system arrangement is shown in Fig. 2.

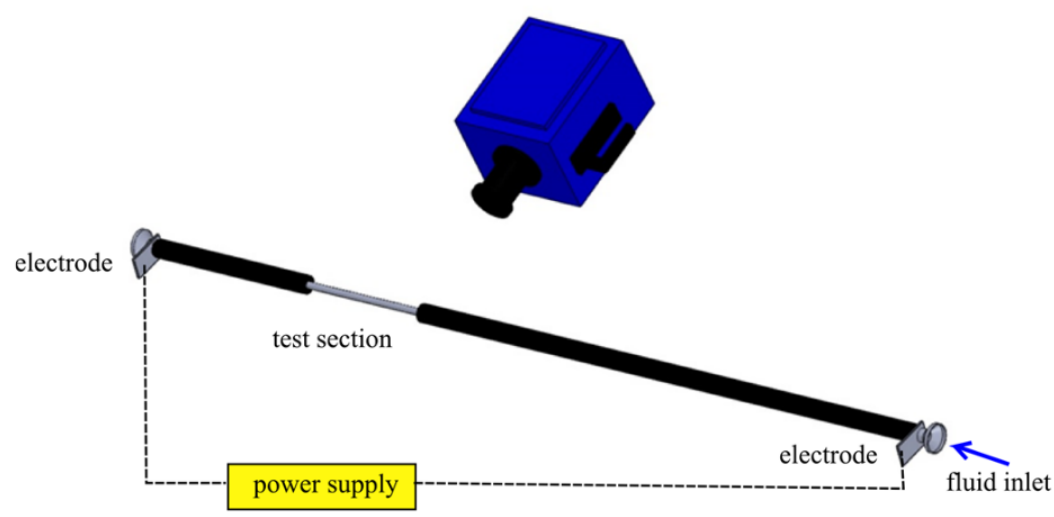

Fig. 2: Sketch of a portion of the experimental setup.

To measure the temperature distribution on the whole test section surface, six images were acquired, moving the infrared camera around the tube's axis. The camera was conveniently fixed on a support, in order to keep the optical axis normal to the tube axis and minimizing perspective artifacts. In the present experimental setup, thanks to limiting the viewing angle to less than $\pm 30^{\circ}$, the surface was considered as a diffuse grey emitter [7]. The effective emissivity of the coating was estimated in situ by shooting a target at different known temperatures, and the value 0.99 was found.

The image processing procedure was made complex by the fact that the observed target surface is not flat: common photo-plans can be produced from images of planar objects by image processing tools based on well-known equations of central projection, but this is not really an easy task when dealing with curved objects [8]. The image processing procedure adopted in this paper was presented in [9]. It permits to rectify optical deformations of the collected images caused by surface curvature: the acquired images were unwrapped and, thanks to adequate position references fixed on the tube wall, were cropped and merged together to obtain continuous temperature map on the tube wall. 


\section{Estimation procedure}

Starting from the temperature distribution of the external wall surface of the tube, it was possible to estimate the local convective heat flux at the fluid-internal wall interface by solving the IHCP by the Tikhonov regularisation method.

The tube wall of the test section was modelled as a 3-D solid domain (sketched in Fig. 3) and the heat conduction problem was written in the discrete domain as follows:

$$
\mathbf{T}=\mathbf{X q}+\mathbf{T}_{\mathbf{q}=\mathbf{0}}
$$

where $\mathbf{T}$ is the vector of the discrete temperature data at the external pipe surface, $\mathbf{q}$ is the heat flux vector at the fluid-internal wall interface, $\mathbf{T}_{\mathrm{q}=0}$ is a constant term and $\mathbf{X}$ is the sensitivity matrix. The sensitivity matrix $\mathbf{X}$ and the term $\mathbf{T}_{\mathrm{q}=0}$ values, for the problem under test, can be calculated numerically [10].

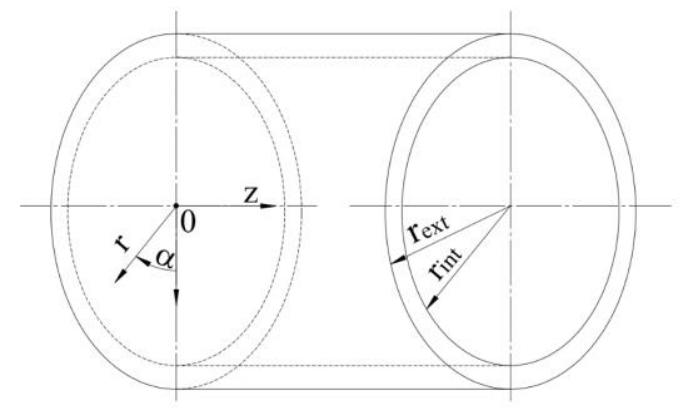

Fig. 3: Geometrical domain with coordinate system.

In the inverse formulation, this computed temperature distribution $\mathbf{T}$ is forced to match the experimental temperature distribution $\mathbf{Y}$ by tuning the convective heat-flux distribution on the internal wall side $\mathbf{q}$. The matching of the two temperature distributions (the computed and the experimentally acquired) could be easily performed under a least square approach. However, due to the ill-posed nature of the problem, the least square solution is generally dominated by noise, and some type of regularisation is required. As mentioned before, in this work the Tikhonov regularisation method is adopted; this approach makes it possible to reformulate the original problem as a well-posed problem that consists of minimising the following objective function:

$$
f(\boldsymbol{q})=\left\|\mathbf{Y}-\mathbf{X q}-\mathbf{T}_{\mathbf{q}=\mathbf{0}}\right\|_{2}^{2}+\lambda^{2}\|\mathbf{L q}\|_{2}^{2}
$$

Where $\|\quad\|_{2}^{2}$ stands for the square of the 2-norm, $\lambda$ is the regularisation parameter, $L$ is the derivative operator and $\mathbf{T}$ is the distribution of the external surface temperature derived from a direct numerical solution of the problem obtained by imposing a given convective heat flux distribution on the internal wall side $\mathbf{q}$. The function expressed in Eq. (2) represents a trade-off between two optimisation processes: first, the fidelity of the fit and second, the smoothness or the stability of the solution. Choosing a large regularisation parameter means that imposing too much regularisation on the solution prejudices the fitting of the data and the ability to obtain a great residual; the absence of regularisation or an insufficiently small regularisation parameter will provide a good fitting but also a solution affected by data errors. Therefore, the choice of a proper regularisation parameter requires a good balance between the size of the residual norm and the size of the solution norm; this it was performed, in the present work, by the discrepancy principle [11]. The discrepancy principle suggests computing $\lambda$ in such a way that the corresponding Tikhonov solution, that is the minimum of the Eq. (2), satisfies the non-linear equation:

$$
\frac{\left\|\boldsymbol{Y}-\left(\boldsymbol{X} \boldsymbol{q}+\boldsymbol{T}_{q=0}\right)\right\|_{2}}{\sqrt{N}}=\sigma_{Y}
$$

where $N$ is the size of the vector $Y$ and $\sigma_{Y}$ is the standard deviation of the measurement error which may be estimated by measuring the surface temperature distribution while maintaining the pipe wall under isothermal conditions. 


\section{Results}

The estimation procedure described above was repeated for different Reynolds number values in turbulent flow regime in the range $5000<\mathrm{Re}<12000$ in order to investigate the effect of the insert on the heat transfer (e.g. local peaks, stagnation points, influence length).

In Fig. 4 a representative temperature map is reported; it highlights the distortion of the wall temperature distribution caused by the presence of the insert device. The centre of the insert, is located at $z \approx 0.03 \mathrm{~m}$.

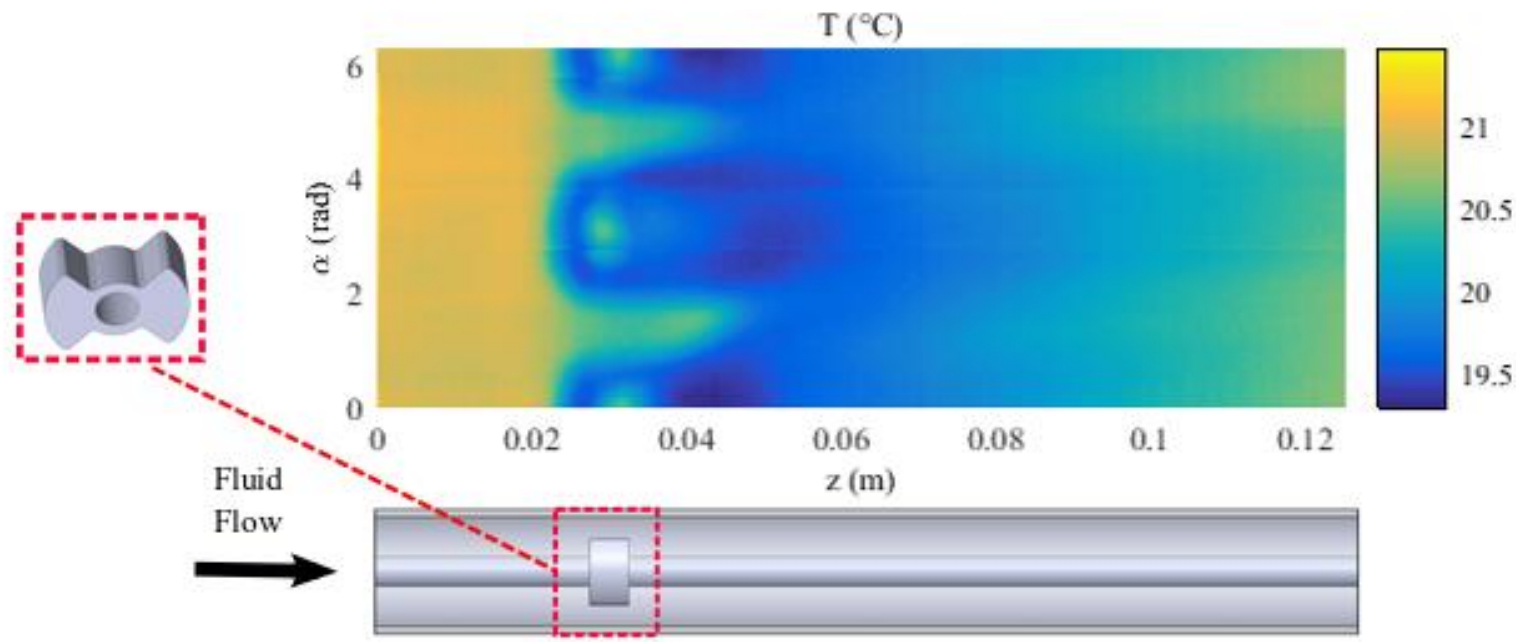

Fig. 4: Wall temperature map $(\mathrm{Re}=8800)$

Before examining the local convective heat transfer characteristics of the tubes fitted with the turbulators, it was investigated how these devices impact on the overall thermal performance of the pipes under test. The heat transfer performances were quantified by means of the Nusselt number, computed as follows:

$$
N u=\frac{h \cdot D_{i n t}}{k_{f l}}
$$

where $k_{f l}$ is the fluid thermal conductivity and $h$ represents the convective heat transfer coefficient averaged over the influence area of the turbulator (i.e., from one tube diameter length before the insert to four diameter lengths after). It was computed as follow:

$$
h=\frac{\bar{q}}{\left(\overline{T-\bar{T}_{b}}\right)}
$$

where $\bar{q}$ and $\bar{T}$ represent the values, averaged over the influence area, of the heat flux and the wall temperature, respectively. $\overline{T_{b}}$ is the average fluid bulk temperature in correspondence of the influence area.

In Fig. 5 it is reported the Nusselt number vs the Reynolds number together with the traditional correlation by Dittus-Boelter [12] for turbulent regime in straight tubes. These data show a significant increment of the Nusselt number over the behavior of straight smooth wall pipe without inserts confirming the goodness of this type of devices as convective heat transfer enhancement technique. 




Fig. 5: Nusselt number distribution vs Reynolds number.

A further insight into the phenomena correlated to the heat transfer rate augmentation was found in the analysis of the local heat flux distribution in correspondence of the insert. From the restored heat flux distribution, it was computed the local heat flux efficiency $\eta$ defined as the ratio between the local heat flux and a reference heat flux value:

$$
\eta=\frac{q_{\text {int }}}{q_{0}}
$$

where $q_{0}$ is the heat flux for the tube, in the same experimental conditions, without the inserts. The maps of this parameter are fundamental to investigate the effect of the inserts in terms of local peaks, stagnation points and influence lengths.

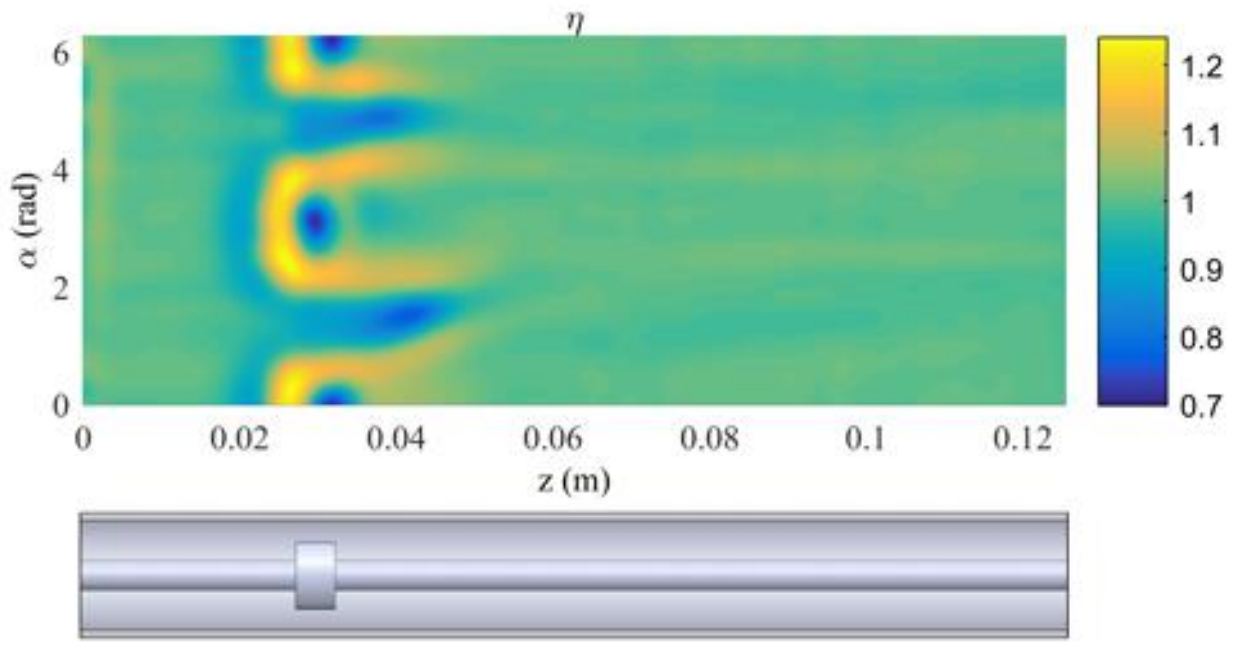

Fig. 6: Heat flux efficiency $\eta(\mathrm{Re}=8800)$. 
In Fig.6 it is reported the local heat flux efficiency distribution for the case of $R e=8800$ : from these data it is possible to notice that the butterfly-shaped device produces a stagnation point in correspondence of the two extreme parts of the "wings" where the temperature of the fluid reaches the highest values and the heat flux distribution presents its minimum. Moreover, it is possible to see that around the stagnation point there is an increase of heat flux and that it is due to the fluid acceleration. Finally, wakes appear in the downstream region. All these behaviours are similar to the ones observed in case of external flow on cross cylinder [12].

It is also possible to notice that on the wall, by the central part of the indention, there is a local minimum in the heat flux distribution: a further investigation on this unexpected aspect is needed and the introduction of further criteria to evaluate local performance should be considered.

To better analyse in details the effect of the studied devices the circumferential distribution of the local heat flux efficiency $\eta$ for the axial coordinate value $z=0.03$ (i.e. center of the insert) is reported in Fig. 7a in polar coordinates; there are two local minima in correspondence of the two extreme parts of the wings (i.e. $\alpha=0^{\circ}$ and $\alpha=180^{\circ}$ ) and the two local peaks at the side of each wing.

a)



b)

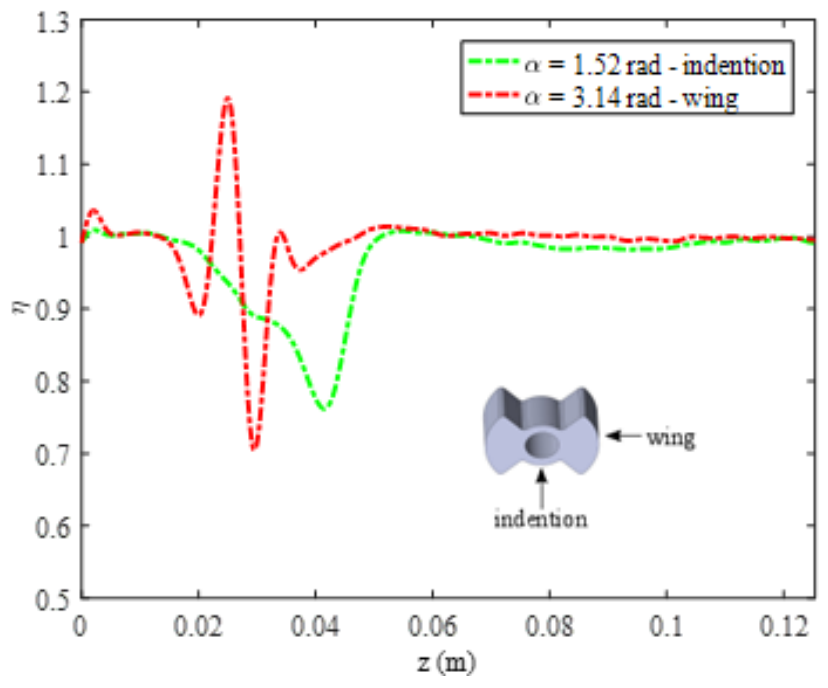

Fig. 7: $\operatorname{Re}=8800$, heat flux efficiency:

a) circumferential distribution for the axial coordinate value $z=0.03$,

b) axial distribution for different angles.

In Fig.7b the axial distribution of the local heat flux efficiency $\eta$ for different values of the angular coordinate $\alpha$ is reported. In particular, there were considered two different distributions, one in correspondence of a "wing" ( $\alpha=3.14$ rad) and one in the central zone of the "indention" of the butterfly shaped insert ( $\alpha=1.52 \mathrm{rad})$. In correspondence of the wing $(z=0.03 \mathrm{~m})$ it is evident the peak of minimum heat flux due to the stagnation point preceded by a local maximum due to 
the fluid acceleration in front and at the side of the wings. The central zone of the indention presents instead a decreasing trend: as first hypothesis, it could be due to a zone of lack of fluid mixing, but further investigations are needed.

In Fig. 8 the local heat flux efficiency distributions is reported for other two representative Reynolds number values.

a)

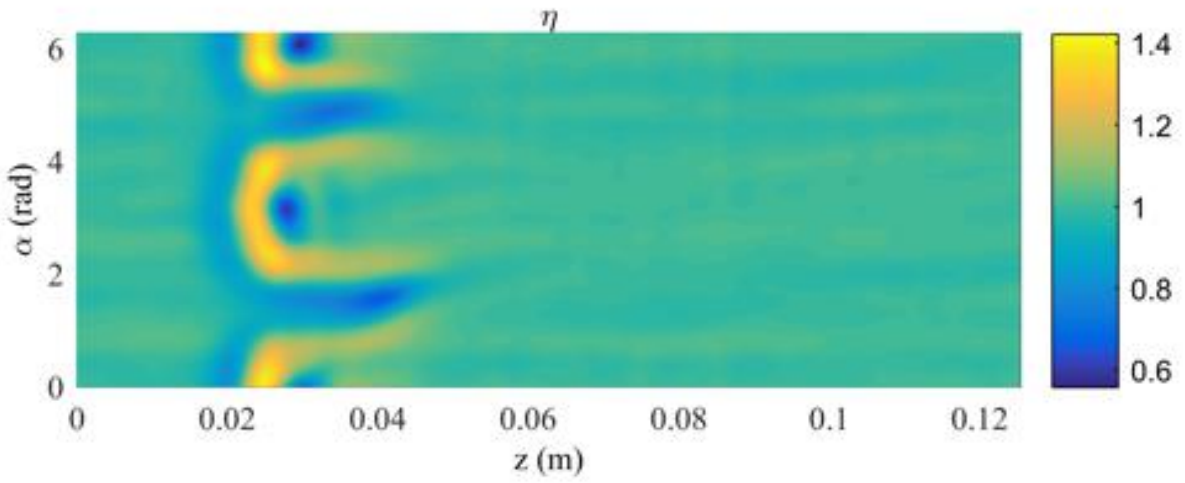

b)

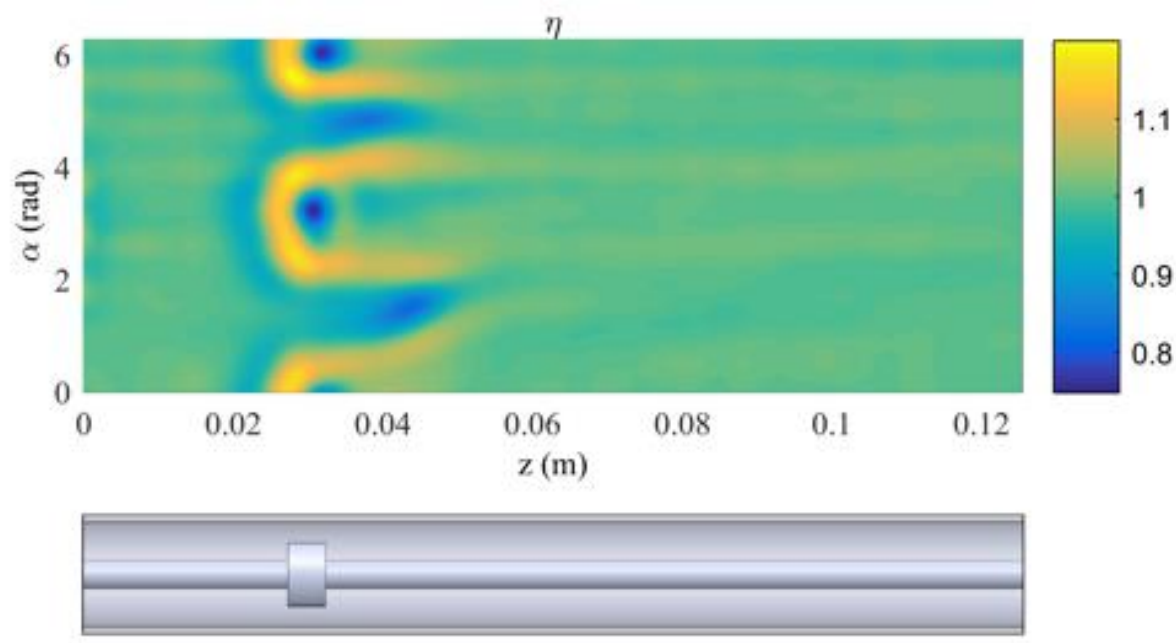

Fig. 8: Restored heat flux efficiency distributions for: a) $\operatorname{Re}=7115$; b) $\operatorname{Re}=11870$

Also for these other two Reynold number values it is possible to recognize the presence of a stagnation point in correspondence of the extreme part of the wings, preceded and followed by local maxima of the heat flux distribution. Moreover, also in these other two cases, there is a local minimum in the heat flux distribution in correspondence of the central zone of the indention.

In Fig. 9 the circumferential distributions of the local heat flux efficiency for the axial coordinate value $z=0.03$ are reported for the same Reynold number values.

Also these graphs show the presence of minimum peaks in correspondence of the extreme parts of the wings enveloped by local maxima of the heat flux distribution. Comparing the $\eta$ circumferential distribution for the three Reynolds number values it could be notice a tendency to smoothen the peaks with the increasing of the Re.

To better highlight this effect, produced by the different Reynolds number values, the axial distributions of the local heat flux efficiency for different angles are shown in Fig. 10. 
a)



b)

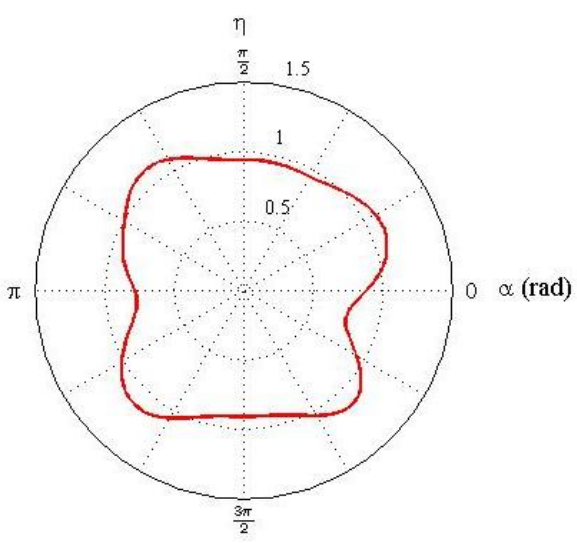

Fig. 9: Circumferential distribution of local heat flux efficiency for the axial coordinate value $z=0.03$ for: a) $\operatorname{Re}=7115$; b) $\operatorname{Re}=11870$

a)

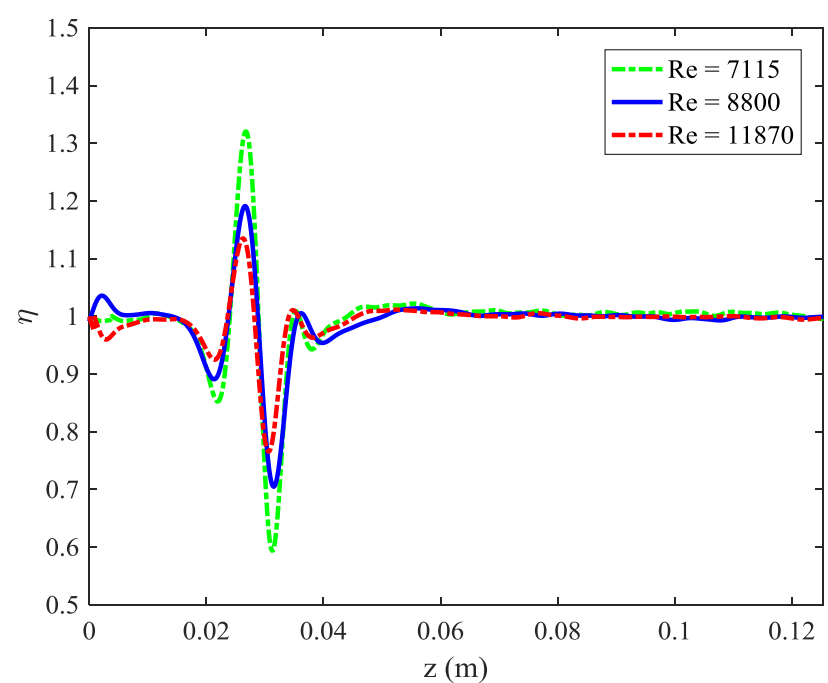

b)

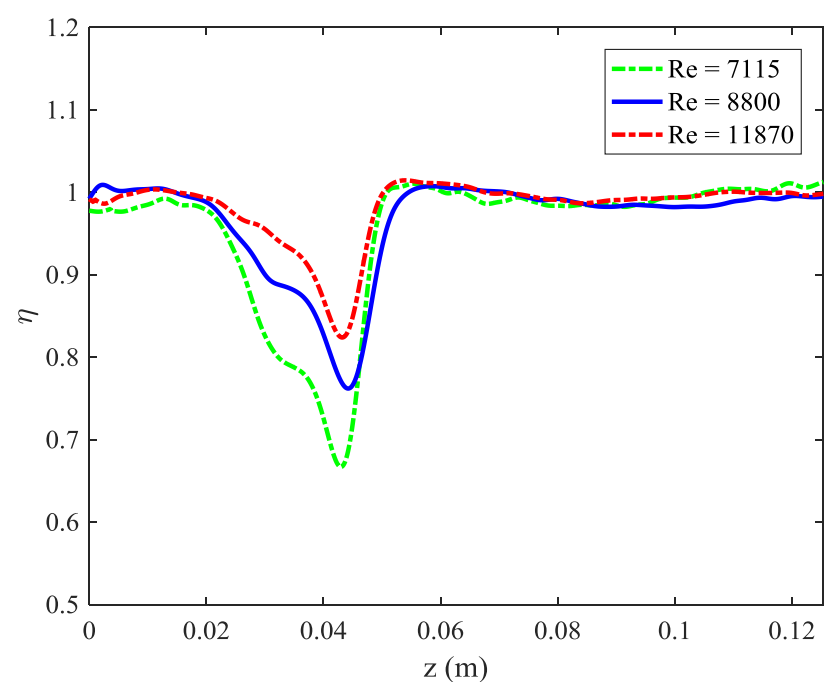

Fig. 10: Axial distribution of local heat flux efficiency for different angles: a) $\alpha=3.14$ rad - wing; b) $\alpha=1.52$ rad indention 
All the three distribution of the local heat flux efficiency reported present similar trends but some differences in the values of the peaks could be observed. In fact, with the increasing of the Reynolds number the peak value decreases and the minimum value increases. In all probability, as observed by Li et al. [13], with increasing of the Reynolds number value, the higher turbulence intensity promotes a more efficient fluid mixing that tends to lessen the difference between the maximum and the minimum values.

\section{Conclusions}

The present work aims to present and test an original experimental procedure for estimating the local convective heat flux in pipes equipped with butterfly-shaped inserts. This kind of investigation is fundamental for this type of insert because, although being effective in the heat transfer enhancement mechanisms, could be critical in some applications due to the presence of great variations in both the temperature and the heat flux distribution. The estimation procedure, based on the filtering approach applied to the solution of the Inverse Heat Conduction Problem within the wall domain, was applied for different Reynolds number values in turbulent flow regime in the range $5000<\operatorname{Re}<12000$. It was observed that the butterfly-shaped device produces a stagnation point in correspondence of the two extreme parts of the "wings" where the heat flux distribution presents its minimum. Moreover, it was possible to notice that around the stagnation point there was an increase of the local heat flux probably due to the fluid acceleration. Finally, wakes appear in the downstream region. All these behaviours are similar to the ones observed in case of external flow on cross cylinder.

The experimental results obtained in the present investigation are particularly useful in the design of innovative heat exchangers equipped with these type of devices. They could be also employed for the validation of numerical models. However, the more important outcome of the present paper is implementing and testing a powerful experimental approach that could be successfully applied for the optimization of the shape of inserts in critical thermal processes.

\section{REFERENCES}

[1] Bergles AE. Handbook of heat transfer. Chapter 11: techniques to enhance heat transfer. New York: McGrawHill;1998.

[2] Dewan, A., Mahanta, P., Sumithra Raju, K. and Suresh Kumar, P., Review of passive heat transfer augmentation techniques. Proceedings of the Institution of Mechanical Engineers, Vol. 218. 2004. 509-527.

[3] Promvonge, P., Heat transfer behaviors in round tubes with conical ring inserts. Energy Conversion and Management, Vol. 49. 2008; 8-15.

[4] Jadooa, A.A., Experimental Investigations Heat Transfer and Pressure Drop Characteristics of Flow Through Circular Tube Fitted with Drilled Cut-Conical Rings. Journal of Engineering and Technology, Vol. 29; 2011. 477487.

[5] Beck, J. V., Blackwell, B., St Clair Jr, C. R., Inverse Heat Conduction: III-Posed Problems, A Wiley-Interscience, New York; 1985.

[6] A.N. Tichonov, V.Y. Arsenin, Solution of III-Posed Problems, Winston \& Sons, Washington; 1977.

[7] Astarita, T. and Carlomagno, G.M., Infrared Thermography for Thermo-fluid-dynamics. Springer, Berlin; 2012.

[8] K. Pavelka, S. Ruzicka, Z. Bila, Photo-Plan Creation of Cylindrical Objects, ISPRS Annals of the Photogrammetry, Remote Sensing and Spatial Information Sciences, Volume II-5/W1; 2013.

[9] Bozzoli, F., Cattani, L. and Rainieri, S., Effect of wall corrugation on local convective heat transfer in coiled tubes. International Journal of Heat and Mass Transfer, Vol. 101; 2016. 76-90.

[10] Bozzoli, F., Cattani, L., Rainieri, S., Bazán, F.S.V, Borges, L.S., Estimation of the local heat-transfer coefficient in the laminar flow regime in coiled tubes by the Tikhonov regularisation method, Int. J. Heat Mass Tran. 72; 2014. 352-61.

[11] Morozov, V.A., Methods for Solving Incorrectly Posed Problems, Springer-Verlag, New York; 1984.

[12] Incoprera F.P., De Witt D.P., Fundamentals of Heat and Mass Transfer, John Wiley \& Sons, Inc. New York; 2002.

[13] Li P., Liu P., Liu Z., Liu W., Experimental and numerical study on the heat transfer and flow performance for the circular tube fitted with drainage inserts, International Journal of Heat and Mass Transfer 107; 2017. 686-696. 